Olga Truba', Aleksandra Rybka', Karolina Klimowicz², Elżbieta M. Grabczak', Małgorzata Żukowska ${ }^{1}$, Marta Dąbrowska', Rafał Krenke'

'Department of Internal Medicine, Pulmonary Diseases and Allergy, Medical University of Warsaw, Warsaw, Poland

'Students' Research Group "Alveolus", Medical University of Warsaw, Warsaw, Poland

${ }^{3} 2^{\text {nd }}$ Department of Clinical Radiology, Medical University of Warsaw, Warsaw, Poland

\title{
Is a normal chest radiograph sufficient to exclude pulmonary abnormalities potentially associated with chronic cough?
}

The authors declare no financial disclosure

\begin{abstract}
Introduction: There are few original studies on the true role of normal chest radiograph (CXR) in exclusion of pulmonary conditions that may be associated with chronic cough. Thus, the aim of the study was to assess whether a plain CXR is a sufficient tool to exclude relevant pulmonary causes of chronic cough.

Material and methods: A retrospective analysis of chest computed tomography (CT) scans in non-smoking patients with chronic cough and normal CXR was performed. The percentage of individuals in whom chest CT revealed relevant abnormalities was compared with the percentage of patients with irrelevant findings or normal chest CT scans. The negative predictive value (NPV) of the CXR in diagnosing the causes of chronic cough was calculated as a proportion of true negative CXRs (normal CXR AND irrelevant CT findings OR normal CT scan) to all negative CXRs (all patients, who had both a CXR and CT scan).

Results: The study group consisted of 59 adult patients with chronic cough, normal CXR and CT scan performed to diagnose the cause of chronic cough. In 21 patients $(21 / 59,35.6 \%)$, chest CT revealed abnormalities that were classified as relevant to chronic cough. The most frequent were: bronchiectasis $(7 / 59,11.9 \%)$, bronchial wall thickening $(6 / 59,10.2 \%)$ and mediastinal lymphadenopathy $(5 / 59,8.5 \%)$. The NPV of a CXR in diagnosing the causes of chronic cough was $64 \%$.

Conclusions: In conclusion, the NPV of CXR in diagnosing pulmonary causes of chronic cough is relatively low. Thus, plain CXR seems to be insufficient to exclude pulmonary diseases potentially associated with chronic cough.
\end{abstract}

Key words: chest radiograph; computed tomography; chronic cough; lung imaging

Adv Respir Med. 2018; 86: 113-120

\section{Introduction}

Chronic cough, usually defined as lasting over 8 weeks, is a common medical complaint, which can be associated with a wide range of pulmonary and extrapulmonary diseases. In epidemiological surveys supported by the European Respiratory Society, cough was reported in 10\% to $20 \%$ of the worldwide population and was responsible for $10-38 \%$ of referrals to pulmonary specialists [1-4]. An even higher prevalence of chronic cough was demonstrated in women and obese patients. The reduction in health-related quality of life associated with chronic cough is comparable with that caused by severe chronic obstructive pulmonary disease (COPD) [5].

Due to a long list of chronic cough causes and limited specificity of various concomitant symptoms, the causal diagnosis is often challenging. Smoking-related bronchitis or COPD should be considered in all current and ex-smokers presenting with chronic cough. Interestingly, although smoking-related bronchitis is one of the most common causes of chronic cough, it only rarely

Address for correspondence: Olga Truba Department of Internal Medicine, Pulmonary Diseases and Allergy, Medical University of Warsaw, Warsaw, Poland,

e-mail: olga.radlinska@gmail.com

DOI: 10.5603/ARM.2018.0018

Received: 18.04 .2018

Copyright (C) 2018 PTChP

ISSN 2451-4934 
prompts smokers to seek medical assistance [1, 2]. Chest radiograph (CXR) is a critical diagnostic procedure in the initial work-up of patients with chronic cough. It may reveal variety of lesions, including tumors, pulmonary consolidations or interstitial patterns and many others, which can be directly or indirectly related to chronic cough [6]. Hence, all published guidelines on the management of chronic cough underline the diagnostic role of CXR in adult patients $[1,7$, 8]. On the other hand, in a significant proportion of patients with chronic cough, CXR does not show any abnormal findings. This scenario also provides relevant diagnostic information, pointing to the need for further differential diagnostics. This should include entities which are known to be the most common causes of chronic cough in non-smoking patients with a normal CXR, i.e. upper airway cough syndrome (UACS) comprising chronic rhinitis, rhinosinusitis; gastroesophageal reflux disease (GERD); asthma and nonasthmatic eosinophilic bronchitis (NAEB) [9].

Even though the role of CXR in the diagnostic algorithm for patients with chronic cough is indisputable, some limitations of this technique should also be recognized. This refers to its relatively low diagnostic sensitivity and specificity compared to other imaging methods, including, in particular, chest computed tomography (CT). It has been demonstrated that CT is more accurate than CXR in diagnosing some pulmonary diseases that may be associated with cough, including interstitial lung diseases or bronchiectasis [10-13]. In the population of children, chest CT is recommended in diagnosing the causes of chronic productive cough [14], while in adults, chest CT is recommended only if the most common causes of cough are excluded $[1,7,8]$. Thus, as the results of management of chronic cough in adults are still unsatisfactory [15], it might be supposed that a wider application of chest CT in this population could improve the efficacy of treatment.

To the authors' knowledge there are no studies directly comparing the sensitivity or accuracy of CXR and CT in diagnosing the causes of chronic cough in adults. Therefore, we decided to perform a study assessing whether the chest radiograph is sufficient to exclude major pulmonary diseases that might be the cause of chronic cough. Chest CT was used as a "gold standard" to calculate the negative predictive value (NPV) of CXR in adult patients with chronic cough.

\section{Material and methods}

\section{Study design}

The study was a retrospective analysis of the results of chest imaging studies performed in patients referred to our institution due to chronic cough between 2010 and 2014.

\section{Patients}

Patients between 18 and 80 years of age were selected from an electronic database of nonsmoking people with chronic cough who were referred to our department in order to diagnose the cause of the condition. All subjects underwent a diagnostic work-up according to the algorithm described elsewhere [9, 16]. The study included patients in whom both CXR and chest CT scan were performed during the diagnostic work-up and CXR did not reveal any abnormalities. Chest CT scans were ordered only in individuals in whom the most common causes of chronic cough (asthma, GERD, UACS or NAEB) were excluded.

\section{Methods}

Chronic cough was defined as cough lasting over 8 weeks $[1,7,8]$. All clinical data were obtained from hospital medical records. The analysis of the imaging studies was based on radiological reports that were prepared by a radiologist with 25 years of experience in pulmonary imaging. All reports were further re-evaluated by one of the authors (OT or AR) in order to confirm and classify if the abnormal findings were relevant to chronic cough.

All evaluated CXRs were digital radiographs performed in posteroanterior projection with the use of automatic control of exposure (Philips Healthcare, DigitalDiagnost, Release 4.1.2). The main system characteristics were as follows: tube current exposure time $1.5 \mathrm{mAs}$, voltage 125 $\mathrm{kV}$, antiscatter grid, a focus to detector distance $150 \mathrm{~cm}$.

The referral to chest CT scanning, as a part of the diagnostic algorithm of chronic cough, was at the discretion of an attending physician. Chest CT scans were performed with a16-row CT scanner (LightSpeed 16 General Electric, USA) using: $1.25-\mathrm{mm}$ collimation, $140 \mathrm{kV}$ peak, 100-250 mA current and matrix size $512 \times 512$. The CT image data were reconstructed with a high spatial frequency algorithm and analyzed in pulmonary window at a window level of $-500 \mathrm{HU}$ and a window width of $1400 \mathrm{HU}$ and mediastinal window at a window level of $40 \mathrm{HU}$ and a window width of $350 \mathrm{HU}$. 
We used a simple classification of chest CT results that included the three following categories: (1) normal chest CT scan, (2) findings relevant and (3) irrelevant to chronic cough. The selection of chest CT findings classified as relevant or irrelevant to chronic cough was based on literature data and the authors' experience. Thus, bronchiectasis, interstitial abnormalities, solitary pulmonary nodules $\geq 8 \mathrm{~mm}$ and significant mediastinal lymphadenopathy (diameter $>10 \mathrm{~mm}$ ) were construed as abnormalities that might have been relevant to chronic cough. All these findings were reported as potentially related to chronic cough in previously published original papers or recommendations [7, 17-20]. The potential role of other CT findings was discussed by all the authors, and a consensus was achieved on classification of the abnormalities. The following chest CT findings were considered to be potentially related to chronic cough by consensus of all the authors: hiatal hernia, bronchial wall thickenings, tree-in-bud pattern and pulmonary embolism. This was because hiatal hernia is a known factor predisposing to GERD while bronchial wall thickening and tree -in-bud sign may represent chronic bronchitis or bronchiolitis, all of which may be related to chronic cough. The latter was reported in $23 \%$ of patients with pulmonary embolism, although it was rather acute than chronic [21]. According to the authors' consensus, the following chest CT findings were classified as probably irrelevant to chronic cough: air trapping, apical scarring of the lungs, atrophic thymus, bullae, calcifications in the mediastinal lymph nodes, small focal band-like parenchymal scars, solitary pulmonary nodules with benign pattern of calcification, small $(<8 \mathrm{~mm})$ solitary pulmonary nodules without calcification, small pleural thickening. Patients with two or more different CT abnormalities classified as relevant and irrelevant, were construed as having pulmonary lesions relevant to chronic cough.

\section{Statistical analysis}

Data are expressed as medians and ranges or numbers and percentages. The NPV of CXR in diagnosing the causes of chronic cough was calculated as a percentage of true negative CXRs (both CXR and CT without any relevant abnormalities) to all negative CXRs (all patients who had both CXR and CT performed). Patients with true and false negative CXRwere compared using Chi-squared test for categorical variables and Mann Whitney U test for continuous variables.

\section{Results}

One hundred and fifty six non-smoking patients with normal CXR were initially selected. In 97 (62\%) of these patients, CXR was the only chest imaging method used to diagnose the cause of chronic cough. In the remaining 59 (38\%) patients, both CXR and chest CT scan were done. These 59 patients formed the proper study group and their clinical and radiological data were submitted for further analysis.

The median age of the study group was 62 years (range $24-78$ years) and $75 \%$ of them were women. The median duration of cough was 54 months (range 3-240 months). Thirty three (56\%) patients had never smoked cigarettes and 26 (44\%) were ex-smokers. The median exposure to tobacco smoke in ex-smokers was 16.5 (range 5-45) pack-years.

In all 59 patients, digital chest radiographs and radiological reports were available for analysis.

Only in 3 of 59 (5\%) subjects, chest CT scan showed completely normal appearance of the chest wall, lung and mediastinal structures. In the remaining 56 (95\%) patients with normal chest radiograph, CT scan revealed some abnormalities.

In $21 / 59$ (36\%) patients, chest CT scans demonstrated at least one lesion possibly related to chronic cough. The most common findings were the following: bronchiectasis $(7 / 59,11.9 \%)$, bronchial wall thickening $(6 / 59,10.2 \%)$ and mediastinal lymphadenopathy $(5 / 59,8.5 \%)$. The detailed data on chest CT findings potentially related to chronic cough are presented in Figure 1 (distribution of relevant abnormalities in chest CT scans [total number of lesions $=26]$ ). In four patients, at least two relevant abnormalities in chest CT coexisted. These were: bronchial wall thickening and tree-in-bud pattern in the first patient, interstitial abnormalities, bronchial wall thickening and mediastinal lymphadenopathy in the second one, bronchial wall thickening and mediastinal lymphadenopathy in the third one and bronchiectasis and diffuse bronchial wall thickening in the fourth patient.

In 35 of 59 patients (59\%), CT demonstrated only nonspecific changes that were classified as irrelevant to chronic cough. Various unconnected abnormalities frequently coexisted. The total number of irrelevant abnormalities demonstrated in 35 patients was 102. The most common irregularities identified in the patients were as follows: small focal band-like parenchymal scars (29/59, $49.2 \%$ ), small (diameter $<8 \mathrm{~mm}$ ) calcified solitary 


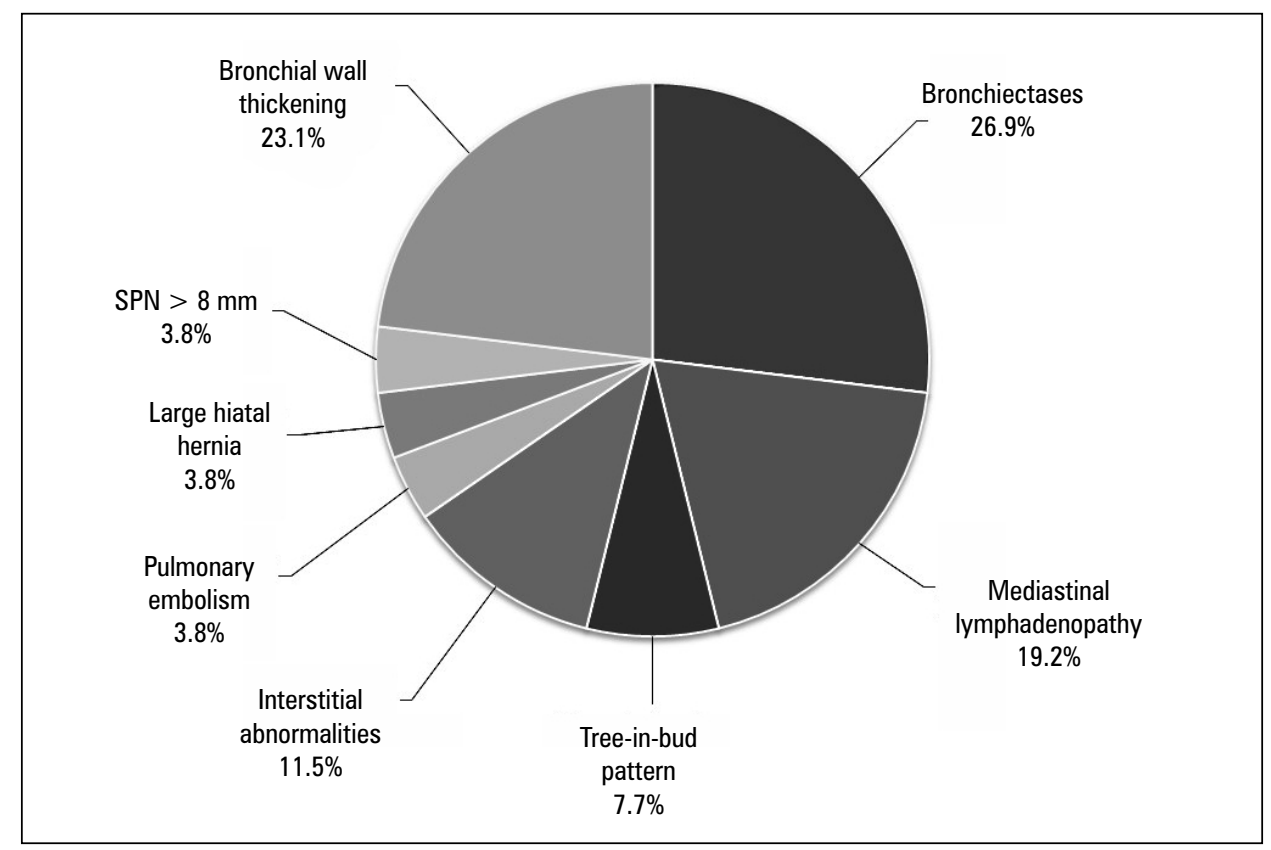

Figure 1. Distribution of relevant abnormalities in chest CT scans (total number of lesions $=26$ )

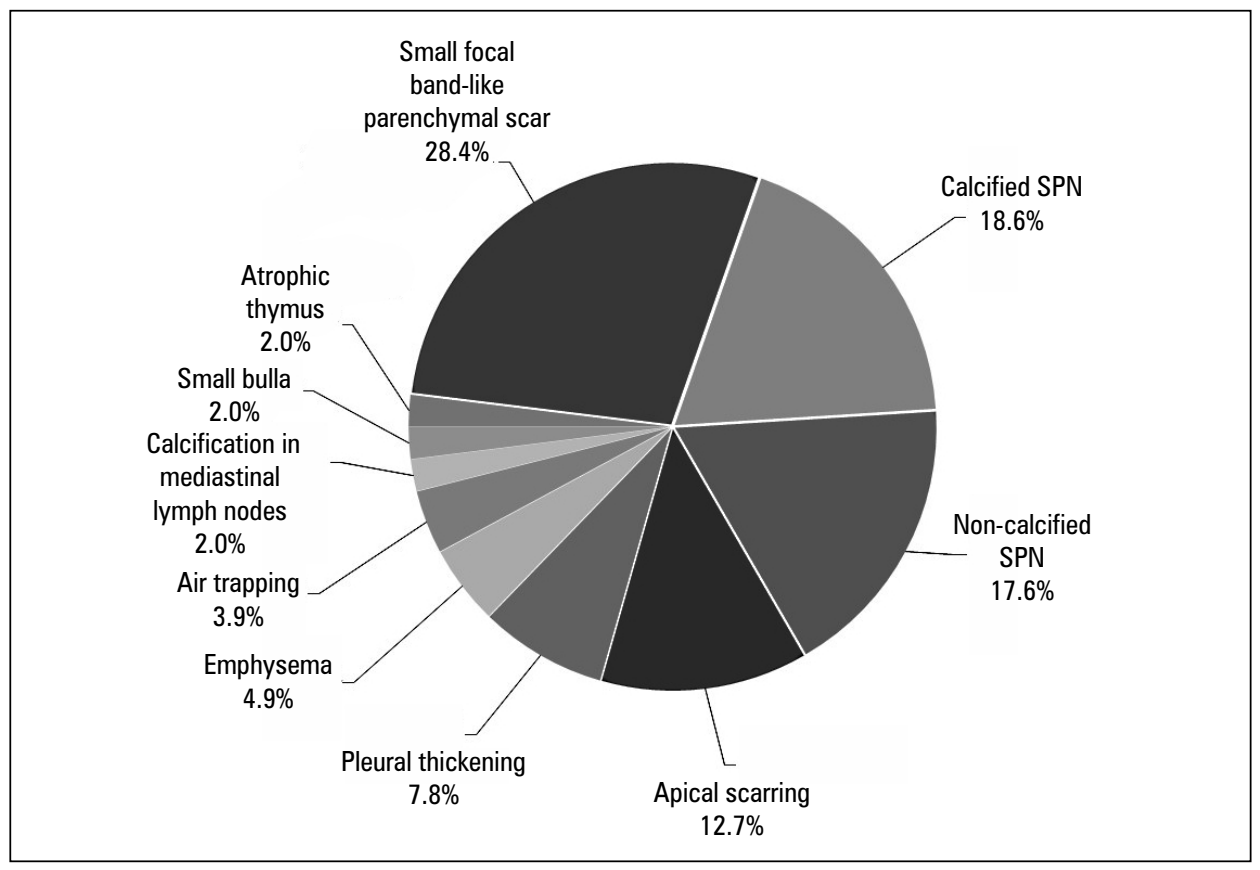

Figure 2. Distribution of irrelevant abnormalities in chest CT scans (total number of lesions $=102$ ); SPN: solitary pulmonary nodule

pulmonary nodules $(19 / 59,32.2 \%)$, small non-calcified solitary pulmonary nodules (range of diameter $<8 \mathrm{~mm})(18 / 59,30.5 \%)$ and apical scarring of the lungs (13/59, 22.0\%). The distribution of all chest CT findings assumed irrelevant to chronic cough are shown in Figure 2. The detailed data on the proportions of relevant and irrelevant CT findings are shown in Table 1.
When comparing patients with true negative and false negative CXR, the duration of cough was shorter in the latter (25 vs 84 months, $\mathrm{p}=0.025$ ). The two groups did not differ as far as age, gender or smoking history were concerned (Table 2).

The NPV of a CXR in diagnosis of relevant causes of chronic cough was calculated as $64 \%$ $(38 / 59)$. 


\begin{tabular}{|c|c|c|c|}
\hline & & $\begin{array}{c}\text { Proportion in relation } \\
\text { to number of patients } \\
n / 59[\%]\end{array}$ & $\begin{array}{l}\text { Proportion in relation to all } \\
\text { relevant abnormalities } \\
n / 26[\%]\end{array}$ \\
\hline \multirow{9}{*}{$\begin{array}{l}\text { CT findings } \\
\text { relevant to chronic } \\
\text { cough }\end{array}$} & Bronchiectases & 11.9 & 26.9 \\
\hline & Bronchial wall thickening without bronchiectases & 10.2 & 23.1 \\
\hline & Mediastinal lymphadenopathy & 8.5 & 19.2 \\
\hline & Interstitial abnormalities & 5.1 & 11.5 \\
\hline & Tree-in-bud pattern & 3.4 & 7.7 \\
\hline & Solitary pulmonary nodule $\geq 8 \mathrm{~mm}$ & 1.7 & 3.8 \\
\hline & Pulmonary embolism & 1.7 & 3.8 \\
\hline & Hiatal hernia & 1.7 & 3.8 \\
\hline & & & $\begin{array}{c}\text { Proportion in relation } \\
\text { to all irrelevant abnormalities } \\
\mathrm{n} / 102[\%]\end{array}$ \\
\hline \multirow{10}{*}{$\begin{array}{l}\text { CT findings } \\
\text { irrelevant to } \\
\text { chronic cough }\end{array}$} & Small focal bandlike parenchymal scar & 49.2 & 28.4 \\
\hline & Calcified SPN & 32.2 & 18.6 \\
\hline & Non-calcified SPN $<8 \mathrm{~mm}$ & 30.5 & 17.6 \\
\hline & Apical scarring & 22.0 & 12.7 \\
\hline & Pleural thickening & 13.6 & 7.8 \\
\hline & Emphysema & 8.5 & 4.9 \\
\hline & Small region of air trapping & 6.8 & 3.9 \\
\hline & Calcification in mediastinal lymph nodes & 3.4 & 2.0 \\
\hline & Small bulla & 3.4 & 2.0 \\
\hline & Atrophic thymus & 3.4 & 2.0 \\
\hline
\end{tabular}

Table 2. Comparison of demographics of patients with true negative CXR and false negative CXR

\begin{tabular}{cccc}
\hline & True negative CXR & False negative CXR & p value \\
\hline Number of patients & 38 & 21 & $\mathrm{~ns}$ \\
Age [yrs] & $61(24-78)$ & $63(31-74)$ & $\mathrm{ns}$ \\
Sex [F/M] & $30 / 8$ & $14 / 7$ & 0.025 \\
Duration of cough [months] & $84(5-192)$ & $25(3-240)$ & $\mathrm{ns}$ \\
Smoking status [NS/ES] & $23 / 15$ & $10 / 11$ & $\mathrm{~ns}$ \\
Smoking history [pack-years] & $19.5(5-30)$ & $12.5(5-45)$ & \\
\hline
\end{tabular}

Data are given as median and ranges or number of patients. Both groups were compared using Chi-squared test for categorical variables and Mann Whitney U test for continuous variables; F: female; M: male; NS: never smoker; ES: ex-smoker

\section{Discussion}

Our study demonstrated relatively low NPV of CXR (64\%) in diagnosing pulmonary diseases that might be associated with chronic cough. This suggests that normal CXR is not sensitive enough to exclude some relevant pulmonary diseases that may be the cause of chronic cough. Given the scarcity of the reliable comparative data on the diagnostic accuracy of CXR vs chest CT in patients with chronic cough, our study may have significant implications for management of patients with chronic cough. It indicates the need for well-designed, prospective studies on the role of CXR in diagnostic algorithm of chronic cough.

Although at first glance it may seem that the results of our study are in conflict with opinions 
presented in various international documents on diagnosis and management of chronic cough, we believe it is the opposite. Guidelines of the British Thoracic Society (BTS), European Respiratory Society (ERS) and American College of Chest Physicians (ACCP) emphasize the role of CXR and spirometry as key and mandatory examinations in patients with chronic cough [1, 7, 8]. According to the above mentioned guidelines, chest CT is recommended only in those patients in whom the cause of cough remains unknown after the initial evaluation $[1,7,8]$. The authors of the ERS guidelines state that the addition of high-resolution chest CT scanning to the baseline investigations in patients with chronic cough is unlikely to be cost-effective [8]. Likewise, the ACCP document recommends that chest CT should be considered only in those patients in whom the most common causes of chronic cough were excluded [7].

It should be emphasized that in terms of chest CT application in the diagnostic work-up of patients with chronic cough our study was fully concordant with the above guidelines. Chest CT scanning was applied only in carefully selected patients in whom the most common causes of chronic cough had been excluded. Therefore, the characteristics of our investigated group (i.e. non-smoking patients in whom asthma, GERD, UACS and NAEB were excluded) is important when interpreting the results and discussing their impact on the management of chronic cough.

The role of chest CT scanning in the diagnosis of various pulmonary diseases is well established. These include, among others, bronchiectasis and idiopathic pulmonary fibrosis - diseases in which the diagnosis is based on CT rather than CXR [7, 21, 22]. It has also been demonstrated that CT may be more useful than CXR in making the diagnosis of other uncommon pulmonary conditions presenting with chronic cough [23]. On the other hand, it should be realized that our knowledge on the role of chest CT scanning in diagnosing patients with chronic cough is based on few studies only. These include one retrospective survey on the diagnosis and management of chronic cough in a general chest clinic, one prospective study reporting the efficacy of CT in the diagnosis of chronic cough causes and one recent study in children [24-26]. The first of these studies was a retrospective analysis of medical records of 124 adult patients, including smokers, presenting with cough lasting for at least three weeks. The authors found that CXR was helpful in establishing the causative factor of chronic cough in $31 \%$ of the subjects. In the same study, chest CT showed significant pulmonary abnormalities in 59\% of the evaluated patients [24]. Thus, chest CT scan revealed significant pulmonary abnormalities in $28 \%$ of individuals in whom the chest radiograph was not able to demonstrate any pulmonary lesions. This finding is consistent with the result of our study that showed relevant pulmonary abnormalities in $36 \%$ of patients with chronic cough and normal CXR.

The next prospective study included 78 nonsmoking patients aged $>12$ years with cough lasting $\geq 3$ weeks, who had a normal chest radiograph. Chest CT scan was performed in $64(82 \%)$ of patients and allowed to reveal the potential cause of chronic cough in $24 \%$ of the subjects [25]. It should be noted that both of the above discussed studies were performed more than 15 years ago. Since then, the technique of CT scanning has evolved and this development might be responsible for slightly better diagnostic yield of chest CT in our study as compared to earlier studies ( $36 \%$ vs 28 and $24 \%$, respectively).

The third study evaluated the diagnostic role of CXR in cough lasting $>4$ weeks. However, that study included only children (two groups of children with median age 3.1 and 5.2 years, respectively); it also differed from our study in terms of design [26]. The authors evaluated whether some cough pointers (signs, symptoms, chest radiograph and spirometry) can differentiate between specific (requiring specific treatment) and non-specific cough, as well as, predict a spontaneous cough resolution. The negative predictive value of normal CXR for the diagnosis of specific cough was as low as 0.15 . Thus, the NPV reported by the authors was much lower than that found in our study. The difference can be explained by numerous dissimilarities between those two studies, including patient characteristics, chronic cough definition (lasting $>4$ vs. $>8$ weeks), chronic cough causes (a priori exclusion of patients with asthma, GERD, UACS or NAEB in our study), and many others. Nevertheless, the general conclusion about low NPV of CXR to exclude specific cough causes (in the study by Chang et al.) and pulmonary abnormalities that may be associated with chronic cough (in our study) appear to be somewhat similar.

The results of our study seem to be particularly important in the context of increasing evidence of the limited efficacy of chronic cough treatment. Chronic idiopathic cough or cough difficult to treat has been diagnosed in as many as $5-10 \%$ of adult patients with chronic cough [27]. As these conditions are diagnoses of exclusion, 
a thorough diagnostic work-up of patients with chronic cough is essential for proper causal diagnosis. Assuming that chest CT scan may reveal pulmonary abnormalities in $24-36 \%$ of patients with chronic cough and normal chest radiograph, the application of this diagnostic procedure seems to be justified in a significant proportion of individuals with unknown cause of chronic cough. Particular vigilance in analyzing CXR is necessary in patients with chronic cough of shorter duration, as false negative CXR is more common in this group than in patients who cough longer.

We are aware about some significant limitations of our study. Firstly, it was a retrospective analysis that included only a limited number of patients. Secondly, the specific indications for chest CT scan had not been predefined, and the decision whether to perform CT or not was somewhat arbitrary. This might have caused a selection bias. Thirdly, the classification of chest CT findings, which could have been or not responsible for chronic cough was arbitrary and not validated. It must be emphasized, however, that to the authors knowledge no other validated classifications which could have been applied exist. Therefore, the authors had to predefine CT abnormalities relevant vs irrelevant to chronic cough basing on literature data and their own experience. Fourthly, it might be argued that the study on true impact of chest CT on management of chronic cough (compared to CXR only) should include data on how often the result of chest CT changed treatment strategy and whether the added therapy improved the overall treatment efficacy. Although we have to agree with this view, it should be noted, that the study was not aimed at the evaluation of the role of chest CT in management of chronic cough. We do believe, this issue can be assessed in future studies.

\section{Conclusion}

In conclusion, although chest radiograph plays a key role in the diagnostic algorithm of chronic cough, its NPV in diagnosing pulmonary causes of the ailment is relatively low. Thus, chest radiograph seems to be insufficient to exclude pulmonary causes of chronic cough.

\section{Compliance with ethical standards}

All procedures performed in studies involving human participants were in accordance with the ethical standards of the institutional and/or national research committee and with the 1964
Helsinki declaration and its later amendments or comparable ethical standards. Due to retrospective character of the analysis, the Institutional Review Board did not endorse this protocol.

\section{Acknowledgments}

The authors would like to thank Marta Maskey-Warzęchowska MD, PhD for her editorial assistance and manuscript review.

\section{Conflict of interest}

The authors declare no conflict of interest.

\section{References:}

1. Morice AH, McGarvey L, Pavord I, et al. British Thoracic Society Cough Guideline Group. Recommendations for the management of cough in adults. Thorax. 2006; 61 Suppl 1: i1-24, doi: 10.1136/thx.2006.065144, indexed in Pubmed: 16936230.

2. Janson C, Chinn S, Jarvis D, et al. Determinants of cough in young adults participating in the European Community Respiratory Health Survey. Eur Respir J. 2001; 18(4): 647-654, indexed in Pubmed: 11716169.

3. Ford AC, Forman D, Moayyedi P, et al. Cough in the community: a cross sectional survey and the relationship to gastrointestinal symptoms. Thorax. 2006; 61(11): 975-979, doi: 10.1136/ thx.2006.060087, indexed in Pubmed: 16809412.

4. Smyrnios NA, Irwin RS, Curley FJ, et al. Chronic cough. The spectrum and frequency of causes, key components of the diagnostic evaluation, and outcome of specific therapy. Am Rev Respir Dis. 1990; 141(3): 640-647, doi: 10.1164/ajrccm/141.3.640, indexed in Pubmed: 2178528.

5. Polley L, Yaman N, Heaney L, et al. Impact of Cough Across Different Chronic Respiratory Diseases. Chest. 2008; 134(2): 295-302, doi: 10.1378/chest.07-0141.

6. Irwin RS, Madison JM. The diagnosis and treatment of cough. New Engl J Med. 2000; 343(23): 1715-1721.

7. Irwin RS, Baumann MH, Bolser DC, et al. Diagnosis and management of cough executive summary: ACCP evidence-based clinical practice guidelines. Chest. 2006; 129(1 Suppl): 1S-23S, doi: 10.1378/chest.129.1_suppl.1S, indexed in Pubmed: 16428686 .

8. Morice AH, Fontana GA, Sovijarvi ARA, et al. ERS Task Force. The diagnosis and management of chronic cough. Eur Respir J. 2004; 24(3): 481-492, doi: 10.1183/09031936.04.00027804, indexed in Pubmed: 15358710.

9. Dąbrowska M, Grabczak EM, Arcimowicz M, et al. Causes of Chronic Cough in Non-smoking Patients. Adv Exp Med Biol. 2015; 873: 25-33, doi: 10.1007/5584_2015_153, indexed in Pubmed: 26285610 .

10. Lauer MS, Lauer MS. Elements of danger--the case of medical imaging. N Engl J Med. 2009; 361(9): 841-843, doi: 10.1056/ NEJMp0904735, indexed in Pubmed: 19710480.

11. Padley SP, Hansell DM, Flower CD, et al. Comparative accuracy of high resolution computed tomography and chest radiography in the diagnosis of chronic diffuse infiltrative lung disease. Clin Radiol. 1991; 44(4): 222-226, indexed in Pubmed: 1959296.

12. Volpe J, Storto ML, Lee K, et al. High-resolution CT of the lung: determination of the usefulness of CT scans obtained with the patient prone based on plain radiographic findings. AJR Am J Roentgenol. 1997; 169(2): 369-374, doi: 10.2214/ ajr.169.2.9242737, indexed in Pubmed: 9242737.

13. van der Bruggen-Bogaarts BA, van der Bruggen HM, van Waes $\mathrm{PF}$, et al. Screening for bronchiectasis. A comparative study between chest radiography and high-resolution CT. Chest. 1996; 109(3): 608-611, indexed in Pubmed: 8617064.

14. Chang AB, Oppenheimer JJ, Weinberger MM, et al. CHEST 
Expert Cough Panel. Children with chronic wet or productive cough - treatment and investigations: a systematic review. Chest. 2016; 149(1): 120-142, doi: 10.1378/chest.15-2065, indexed in Pubmed: 26757284

15. Chamberlain SAF, Garrod R, Douiri A, et al. The impact of chronic cough: a cross-sectional European survey. Lung. 2015; 193(3): 401-408, doi: 10.1007/s00408-015-9701-2, indexed in Pubmed: 25787221

16. Grabczak EM, Dabrowska M, Krenke R, et al. Does the established cause of chronic cough depend on diagnostic approach? J Physiol Pharmacol. 2008; 59 Suppl 6: 285-296, indexed in Pubmed: 19218652.

17. Kastelik JA, Aziz I, Ojoo JC, et al. Investigation and management of chronic cough using a probability-based algorithm. Eur Respir J. 2005; 25(2): 235-243, doi: 10.1183/09031936.05.00140803, indexed in Pubmed: 15684286

18. Ribeiro M, De Castro Pereira CA, Nery LE, et al. A prospective longitudinal study of clinical characteristics, laboratory findings, diagnostic spectrum and outcomes of specific therapy in adult patients with chronic cough in a general respiratory clinic. Int J Clin Pract. 2006; 60(7): 799-805, indexed in Pubmed: 16858753.

19. Pratter MR. Overview of common causes of chronic cough: ACCP evidence-based clinical practice guidelines. Chest. 2006; 129(1 Suppl): 59S-62S, doi: 10.1378/chest.129.1_suppl.59S, indexed in Pubmed: 16428693.

20. Irwin RS, Boulet LP, Cloutier MM, et al. Managing cough as a defense mechanism and as a symptom. A consensus panel report of the American College of Chest Physicians. Chest. 1998; 114(2 Suppl Managing): 133S-181S, indexed in Pubmed: 9725800 .
21. Konstantinides SV, Torbicki A, Agnelli G, et al. Task force for the diagnosis and management of acute pulmonary embolism of the european society of cardiology (ESC). 2014 ESC guidelines on the diagnosis and management of acute pulmonary embolism. Eur Heart J. 2014; 35(43): 3033-69, 3069a, doi 10.1093/eurhearti/ehu283, indexed in Pubmed: 25173341.

22. Raghu G, Collard HR, Egan JJ, et al. ATS/ERS/JRS/ALAT Committee on Idiopathic Pulmonary Fibrosis. An official ATS/ERS/ JRS/ALAT statement: idiopathic pulmonary fibrosis: evidence -based guidelines for diagnosis and management. Am J Respir Crit Care Med. 2011; 183(6): 788-824, doi: 10.1164/rccm.2009040GL, indexed in Pubmed: 21471066.

23. O'Beirne SL, Escalon JG, Arkin JE, et al. Chronic cough and bilateral pneumothoraces in a nonsmoker. Chest. 2016; 149(2): e49-e55, doi: 10.1016/j.chest.2015.10.070, indexed in Pubmed: 26867855.

24. McGarvey LP, Heaney LG, MacMahon J. A retrospective survey of diagnosis and management of patients presenting with chronic cough to a general chest clinic. Int J Clin Pract. 1998; 52(3): 158-161, indexed in Pubmed: 9684430.

25. Palombini BC, Villanova CA, Araújo E, et al. A pathogenic triad in chronic cough: asthma, postnasal drip syndrome, and gastroesophageal reflux disease. Chest. 1999; 116(2): 279-284, indexed in Pubmed: 10453852.

26. Chang AB, Van Asperen PP, Glasgow N, et al. Children with chronic cough: when is watchful waiting appropriate? Development of likelihood ratios for assessing children with chronic cough. Chest. 2015; 147(3): 745-753, doi: 10.1378/ chest.14-2155, indexed in Pubmed: 25501672.

27. Gibson P, Wang G, McGarvey L, et al. CHEST Expert Cough Panel, CHEST Expert Cough Panel. Treatment of Unexplained Chronic Cough: CHEST Guideline and Expert Panel Report. Chest. 2016; 149(1): 27-44 